
et MATERIALIA
Vol. 25, pp. 607-612, 1991

Printed in the U.S.A.
Pergamon Press plc

A11 rights reserved

\title{
RELAXED CONFIGURATION OF A ROW OF PUNCHED PRISMATIC DISLOCATION LOOPS
}

\author{
David C. Dunand and Andreas Mortensen \\ Department of Materials Science and Engineering \\ Massachusetts Institute of Technology, Cambridge, MA 02139, USA. \\ (Received December 19, 1990)
}

\section{Introduction}

Punched rows of coaxial, circular prismatic dislocation loops can be produced in a crystal by several mechanisms: growth of a second phase in a ductile matrix (1), growth of gas bubbles in irradiated metals (2), mechanical indentation (3), elastic constant mismatch strains induced by isostatic pressurization (4) or by shear (5) and strain mismatch due to a difference of coefficient of thermal expansion and temperature change $(6,7)$. The loops within such a row are nucleated one by one at their source, from which they are repelled by following loops until an equilibrium configuration is reached where nucleation stops and all loops are at rest, unable to overcome lattice friction. The resulting loop spacings in the row are of interest since they govern the local dislocation density in, as well as the size of, the resulting plastic zone.

Unlike the related problem of a pile-up of straight dislocations of infinite length, there exists no closed-form expression for loop spacing within such a row. From expressions for the elastic stress field surrounding a single loop in an isotropic crystal, Bullough and Newman (8) computed the equilibrium configuration of such a row after making the simplifying assumption that only first and second nearest neighboring loops interact. They considered a row of ten loops for three different values of a dimensionless parameter $v$ which combines the lattice properties and the loop diameter. They noted that their neglect of the interaction of loops separated by more than two neighbors is invalid when loops are closely spaced, i.e., for a large number of loops or a high value of the parameter $v$.

In this paper, we first explore the simpler case where all but nearest neighbors interactions are negligible. This yields a closed-form solution for the problem. We then use this solution as a tool to compute equilibrium configurations for loop numbers and values of the parameter $v$ beyond those explored by Bullough and Newman (8). From the results, we deduce a "best-fit" relationship between the dimensionless length of the row and its total number of loops, which can be used to apply results of our calculations easily. The smallest loop spacing in a row is given to define the range of validity of the calculations. The largest loop spacing in a row is also calculated, to allow measurement of lattice friction stress by microstructural observation of such loop rows. We then calculate the equilibrium configuration of two rows of prismatic loops of opposite Burgers vector placed on opposite sides of their source. This situation can arise when a two-phase material is sheared (9) and is mathematically equivalent to a single row attracted to a free surface. Finally, we give a simple solution for the average backstress exerted on an obstacle by the relaxed row of loops.

\section{Theory}

\section{Equilibrium configuration of loop rows}

Consider a circular prismatic dislocation of diameter $d$ and Burgers vector $b$ lying in the plane $z=0$ with its center at the origin of a cylindrical coordinate system $\rho, \phi$ and $z$. The shear stress on the glide cylinder $\tau_{r z}(\zeta)$ induced by this loop in the infinite, isotropic crystal is given by Kroupa (10) and Bullough and Newman (8) as :

$$
\tau_{r \mathrm{r}}(\zeta)=\frac{b G}{2 \pi d(1-v)} \cdot \frac{\zeta \cdot K\left[\left(\zeta^{2}+1\right)^{-1 / 2}\right] \cdot\left(\zeta-\zeta^{-1}\right) \cdot E\left[\left(\zeta^{2}+1\right)^{-1 / 2}\right]}{\left(\zeta^{2}+1\right)^{3 / 2}},
$$

where $\zeta=z / d, G$ is the shear modulus, $v$ the Poisson's ratio and $E$ and $K$ are complete elliptical integrals. 
Bullough and Newman (8) used Eq. (1) to determine loop spacings in a row of coaxial, prismatic dislocation loops of diameter $d$ in the half space $z \geq 0$, the first loop being fixed and located at the origin. They calculated the dimensionless position $\zeta$ for each loop within a row of ten loops for three different values of the parameter $v$ :

$$
v=\frac{2 \pi d(1-i) \tau_{c}}{b G}
$$

where $\tau_{c}$ is the local critical shear stress (or loop friction stress) needed to move the dislocation constituting the loop against the lattice friction. The equilibrium configuration of such a row is reached when the shear stress on each and every loop (except the last punched loop) due to its neighbors is equal to $\tau_{c}$.

The choice of the number of neighbors to be considered in the stress computation can be made arbitrarily, as did Bullough and Newman (8). Mathematically, all neighboring loops should be considered since the elastic stress field has an infinite reach; this $\mathrm{n}$-body equilibrium problem however leads to prohibitively long calculation times for a large number of loops in the row. As a compromise, we consider only neighbors which exert a stress larger than the critical shear stress on the loop to be equilibrated. We expect this assumption to be at possible fault only at the ends of the row, where the stress fields of distant loops do not cancel one another. When this situation occurs at the blocked end of the row, the relative error is small since nearest neighbor loop spacings are at their smallest. The stress due to nearest neighbors is therefore very large compared to $\tau_{c}$. At the other "free" end, loop spacings are generally large, so the stresses due to higher onder neighboring loops is most likely to decay rapidly below the loop friction stress.

A first order approximation is to consider only nearest neighbor interactions. The problem then has a mathematically simple solution, which is most easily derived by considering a physically equivalent and conceptually simpler situation. Consider a row of $n$ identical solid bodies, aligned on a single axis, and only able to move along that axis. Each body is connected to its two nearest neighbors by a non-linear spring (Fig. 1a), which exerts a repulsive force $F_{s}$ :

$$
F_{s}(\Delta \zeta)=\pi d b \tau_{r z}(\Delta \zeta)
$$

where $\tau_{r 2}(\Delta \zeta)$ is given by Eq. (1) and $\Delta \zeta$ is the distance between the body and its neighbor. Motion of each body is opposed by a constant friction force $F_{f}$ :

$$
F_{f}=\pi d b \tau_{c} .
$$

The first body is pushed towards its neighbors until all bodies move at steady state along the axis. Now, consider the $i^{\text {th }}$ body in this row of $n$ bodies, $i=1$ denoting the body on which the external force is applied. The spring between body $i$ and its neighbor $i+1$ - which are separated by a distance $\Delta \zeta_{i}$ - must oppose a force equal to the friction of the $(n-i)$ bodies that are moving ahead of it:

$$
F_{s}\left(\Delta \zeta_{i}\right)=(n-i) \cdot F_{f},
$$

or, after introducing Eqs. (3) and (4) in Eq.(5) :

$$
\tau_{r z}\left(\Delta \zeta_{i}\right)=(n-i) \cdot \tau_{c} .
$$

The spacing is then found by inserting Eq. (1) in Eq. (6) and solving for $\Delta \zeta_{i}$. Since all governing equations are the same, the configuration of the row of moving bodies is identical to that of the row of loops under consideration (Fig. 1b). The position $\zeta_{i}$ of loop $i$, measured from the blocked loop, is :

$$
\zeta_{i}=\sum_{j=1}^{i-1} \Delta \zeta_{j}
$$


The total train length is $\zeta_{n}$. It is noted that the position of loop $i, \zeta_{n}-\zeta_{i}$, measured from the opposite, "free" end of the train, is independent of the total number of loops $n$ in the train. The problem is therefore solved by successive solution of equation (6), the n-body solution being constructed as a superposition of $(n-I)$ two-body solutions.

A computer program was written to find the equilibrium configuration of a row of loops using Eq. (1) and a numerical solution for elliptical integrals given by Abramovitz and Stegun (11). Eq. (6) was used to generate the initial loop positions. The computational algorithm was designed to seek equilibrium positions of each loop in a row, starting with the last "free" one, by changing loop position until the stress to which it is subjected falls in the range $\tau_{c} \pm 0.005 \cdot \tau_{c}$. This iterative procedure was stopped when the equilibrium condition was satisfied for all loops in the row (except the first one), and no loop in the row was moved in the last iteration by both more than $0.5 \%$ of the distance to the origin, and more than an absolute value of $\Delta \zeta=10^{-3}$.

Different initial conditions were also tried to check for chaotic behavior. Results were identical provided that the shear stress on each loop in the initial configuration was larger than the critical shear stress, i.e., provided that each loop was initially submitted to a stress pushing it in the positive direction of the $z$-axis away from the blocked loop. It was also found that the computation time was significantly decreased by using an initial condition based on results from previous computations for $n^{\prime}<n$ for the first $n^{\prime}$ loops, and Eq.(6) for the remaining loops. This initial condition was used in most of this work.

\section{Backstress exerted by a row of loops on their source}

Consider a equilibrated row of $n$ dislocations loops, emanating from a punch or inclusion of second phase material. Imagine that its surface is "greased" so that it can move freely through the crystal while atoms in the vicinity of the interface relocate reversibly by diffusion to allow motion of the inclusion at no energy cost. Suppose now that the inclusion is slowly pushed by a force $F_{b}$ toward the equilibrated row of loops, by a distance dx larger than several interatomic spacings. Since they are at equilibrium, the loops move with the particle, the end-result being a translation by a distance $\mathrm{dx}$ of the entire system particle/row of loops. Since the particle is assumed to move reversibly, the work $d W_{b}=F_{b} d x$ spent in the operation is equal to the energy $d W_{f}=n F_{f} d x$ dissipated by the $n$ loops moving against the friction force $F_{f}$. Assuming that the force on the inclusion induces an average stress $\sigma_{b}=F_{b} / A$ (where $A$ is the cross-sectional area of the inclusion), the average backstress is found by equating $d W_{b}$ and $d W_{f}$ :

$$
\sigma_{b}=\frac{n \tau_{f} b \pi d}{A}
$$

It is noted that, while the form and range of the stress field will determine the spacing of the equilibrated row, it has no influence on the backstress on the inclusion. The above derivation is only valid in an infinite crystal with a single inclusion and row of loops. For closely spaced inclusions with interacting rows of loops, Ashby's derivation (12) of the average backstress should be used. This simple derivation is approximate because changes in the stress state of the inclusion induced by "greasing" the interface (which relaxes interfacial stresses) are neglected. Also, the results say nothing about the local stress distribution inside the inclusion.

We also note that, by similar reasoning, we find the well known result that the force exerted by a pile-up of $n$ dislocations of length $L$ on an obstacle in an applied shear stress field $\tau \gg \tau_{c}$, is $n t b L$.

\section{Results}

While considering all relevant neighbors makes the calculation significantly more lengthy than using the secondnearest neighbor assumption made by Bullough and Newman (8), results differ significantly at small loop spacings. An example for a row of 100 loops is given in Fig. 2 with the parameter $v$ equal to 0.1 and 1 , and compared with those of the nearest neighbor solution (Eq. (6)). It is found that Eq. (6) constitutes an excellent approximation for values of $v$ smaller than 0.1 .

The minimum distance between two loops in a row $\Delta \zeta_{I}$ is found to decrease exponentially with the total number of loops $n$ in the row (Fig. 3). This minimum distance is of interest since it defines the maximum number of loops for which the present calculation is meaningful: since Eq. (1) is based on continuum mechanics, it is only valid for loop spacings much larger than a Burgers vector, i.e. when $\Delta \zeta_{I}$ is much larger than $\mathrm{b} / \mathrm{d}$. 
Fig. 4 displays the length of the row, i.e., the position of the last loop $\zeta_{n}$. It is found that, for the values of $n$ explored and for $0.05 \leq v \leq 5$, the length of a row is closely approximated by a power-law function of the total number of loops:

$$
\zeta_{n}=1.74 v-0.64 n 0.34
$$

The relative error when using this expression is on average $14 \%$, the maximal deviation found being $25 \%$. Thus, if the length and number of loops of a row are known experimentally, the parameter $v$ and therefore the loop friction stress can be found. In practice however, it can be difficult to determine the exact number of loops in a row either because of lack of resolution (as in X-ray topography and decoration techniques) or because the field of view is smaller than the row length (as in transmission electron microscopy). An equally suitable and more convenient parameter to determine $v$ is then the spacing $\Delta \zeta_{n-1}$ between the last and the penultimate loops of a row. This spacing is virtually independent of the total number of loops in the row $n$, but is sensitive to $v$. Curve-fitting yields:

$$
\log v=0.866-1.08 \cdot \Delta \zeta_{n-1}+0.102 \cdot \Delta \zeta_{n-1}^{2}
$$

We now turn to the problem of two rows of prismatic loops, respectively of interstitial atoms and vacancies, placed symmetrically on each side of the plane $z=0$ and prevented from annihilating by locking of the leading loop of each row, held a reduced distance $\Delta \zeta_{o}$ apart (Fig. 1c). This problem has been treated by Head (13) for straight dislocations of infinite length. This configuration describes a row of loops whose first blocked loop is at a distance $\Delta \zeta_{0} * / 2$ of a free surface parallel to the plane of the loops. Alternatively, the double train can arise when, upon shearing of a two-phase material, an inclusion produces two rows of geometrically necessary loops of opposite Burgers vector on each of its sides (9).

The relative difference in loop spacings $\delta_{i}=\left(\Delta \zeta_{i}-\Delta \zeta_{i}^{*}\right) / \Delta \zeta_{i}$ in the undisturbed row $\Delta \zeta_{i}$ and the corresponding spacing in the double row $\Delta \zeta_{i}^{*}$ is shown in Fig. 5 for two values of $v$ and $\Delta \zeta_{0}^{*}$. The disturbance decays more rapidly for the larger value of $v$, since the overall distance from the origin of each loop is larger and thus the attraction of the opposite train is smaller. As expected, the smaller value of $\Delta \zeta_{o}$ leads to a larger disturbance for the first loops of the train and a larger number of loops which are significantly disturbed. The absolute value of the departure is, however, small for all loops, even for the small values of $\Delta \zeta_{0}$. The attractive effect might hardly be noticeable experimentally, except for the very first closely spaced loops of the row. This implies, for example, that the loop friction stress can be measured with adequate precision using Eq. (10), which was derived for a single row of loops in an otherwise stress-free crystal.

\section{Conclusions}

Loop spacing in a row of circular, coaxial, prismatic loops emanating from a single source are calculated accounting for all interacting neighbors. Best-fit equations are given for the total row length and the maximum loop spacing, for the range of dimensionless parameters explored. The maximum loop spacing in a row is found to be independent of the total number of loops the row, but is sensitive to a dimensionless parameter $v$ dependent upon the loop friction stress and the loop diameter.

A closed-form solution is derived for the case where all but first neighbor loop interactions are neglected. This solution agrees well with computed results for $v \leq 0.1$. The backstress of a relaxed row of loops on its source is also derived.

A system of two blocked rows of loops of opposite Burgers vector and direction is examined; it is concluded that the disturbance as compared to an equivalent undisturbed row is small.

Acknowledgments: This project was supported by the National Science Foundation, Grant \# DMR-9002558. Access to project ATHENA's computing facilities at M.I.T. is gratefully acknowledged.

\section{References}

(1) B. J. Makenas, H.K. Bimbaum, Acta Met. 28, 979 (1980). 
(2) P.B. Johnson, A.L. Malcolm, D.J. Mazey, Nature 329, 316 (1987).

(3) Y. Miura, H. Nakamura, K. Kaieda, Jap. Inst. Met. Trans. 17, 793 (1976).

(4) M.F. Ashby, S.H. Gelles, L.E. Tanner, Phil. Mag. 19, 757 (1969).

(5) F.J. Humphrey, P.B. Hirsch, Proc. Roy. Soc. A 318, 73 (1970).

(6) J.W. Mitchell, Growth and Perfection of Crystals, p. 386, J. Wiley, New York (1958).

(7) D.C. Dunand, A. Mortensen, On Plastic Relaxation of Thermal Stresses in Reinforced Metals, accepted Acta Metall. Mater.

(8) R. Bullough, R.C. Newman, Phil. Mag. 8, 5, 921 (1960).

(9) P.B. Hirsch, J. Inst. Met. 86, 7 (1957).

(10) F. Kroupa, Czech. J. Phys. 10, 284 (1960).

(11) M. Abramowitz, I.E. Stegun, Handbook of Mathematical Functions, $10^{\text {th }}$ Printing, p. 591, National Bureau of Standards, Washington D.C. (1972).

(12) M.F. Ashby, Phil. Mag. 14, 1157 (1966), 21, 399 (1970).

(13) A.K. Head, Phil. Mag. 4, 295 (1959).

(a)

(b)

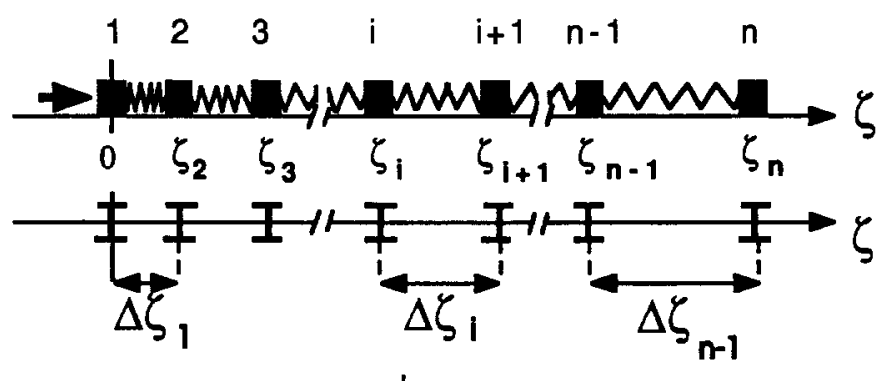

(c)

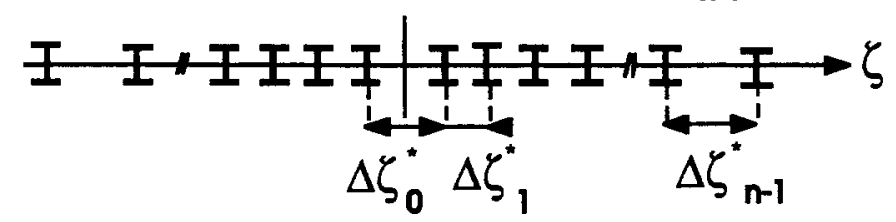

Figure 1: (a) Steady-state configuration of a moving row of $n$ bodies, each submitted to a friction force. (b) Equivalent configuration of a row of $\boldsymbol{n}$ circular, prismatic loops repelling each other in a lattice with a non-zero friction stress (the first loop is blocked). (c) Configuration of two rows of prismatic loops of opposite Burgers vector, located symmetrically on each side of the origin. The first loops of each rows are blocked.

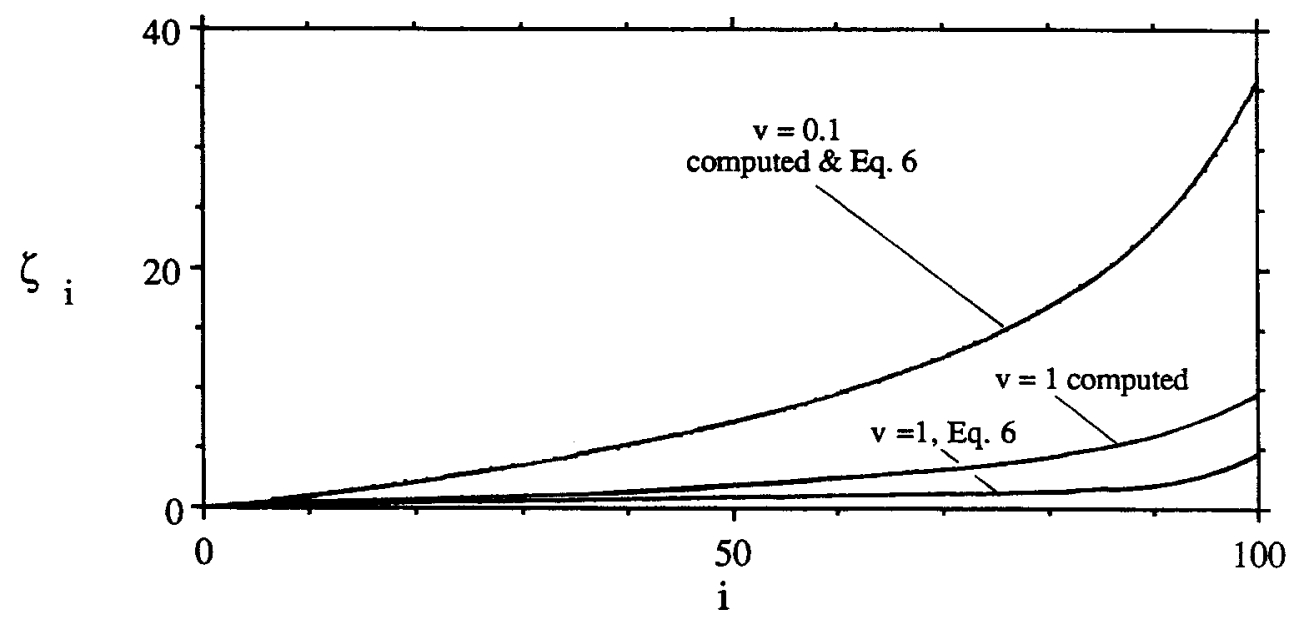

Figure 2: Dimensionless distance from the origin for a row of 100 loops for two values of $v$, as calculated from Eq. (6) and as computed. 


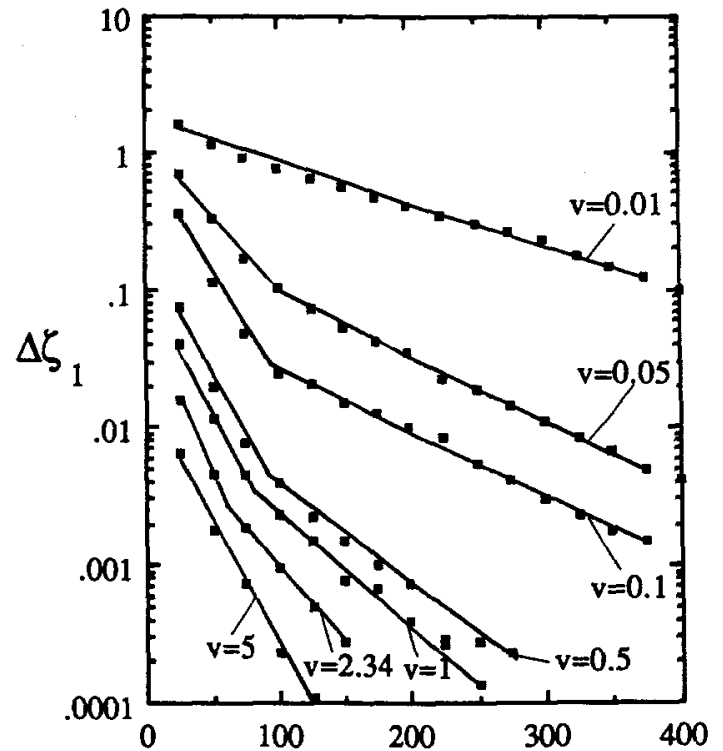

n

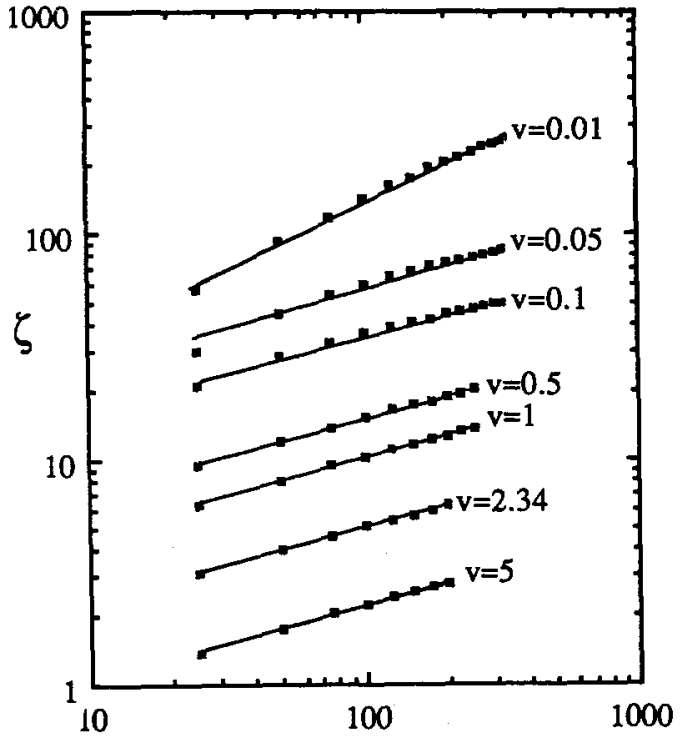

n

Eigure 3: Dimensionless minimum spacing between two loops (first two loops) as a function of the number of loops in the row for different values of $v$.

Figure 4: Dimensionless length of a row as a function of the number of loops for different values of $v$.
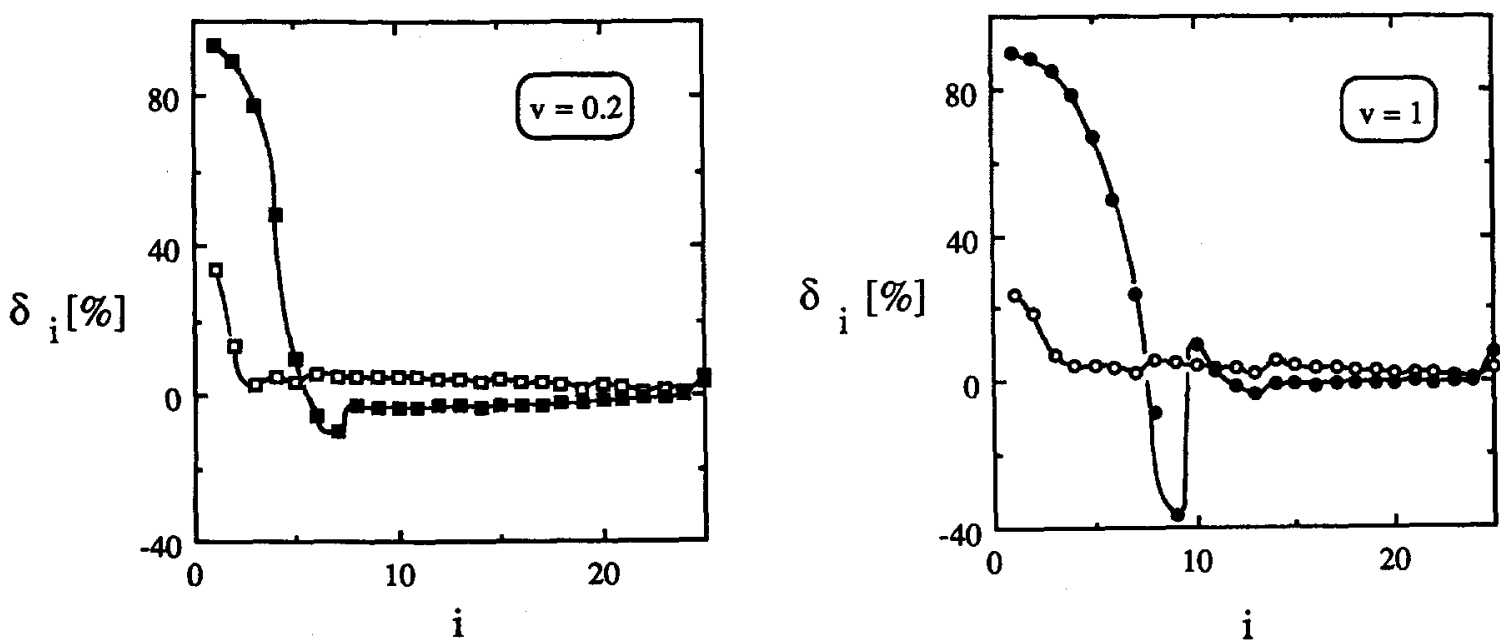

Figure 5: Relative difference in loop spacings between the undisturbed row (Fig. 1b) and the corresponding disturbed row (Fig. 1c, $\zeta>0$ ) for two values of $v$. Empty symbols: $\Delta \zeta_{0}^{*}=1$, full symbols: $\Delta \zeta^{*}=0.1$. 